\title{
INFLUÊNCIA DO ERRO DE ESTIMAÇÃO DO COMPRIMENTO DAS BASELINES NA DETERMINAÇÃO DA ATITUDE GNSS
}

\author{
Heber Valdo Nogueira*, Elder Moreira Hemerly* \\ *Divisão de Engenharia Eletrônica \\ Instituto Tecnológico de Aeronáutica \\ São José dos Campos, São Paulo, Brasil \\ Emails: hebervn@gmail.com, hemerly@ita.br
}

\begin{abstract}
The carrier phase measurements are inevitably necessary in problems involving high accuracy GNSS (Global Navigation Satellite System) positioning. The challenge in using this measurement is to estimate the phase ambiguity. For real time applications, the ambiguities should be solved with the least possible amount of data, but this can affect the accuracy of baselines and therefore attitude. The distance between receivers affect attitude precision. This work shows the influence of the baseline length attitude determination on-the-fly.
\end{abstract}

Keywords - Navigation, Ambiguity Resolution, Baseline.

Resumo- As medidas de fase da onda portadora são, inevitavelmente, necessárias em problemas envolvendo o posicionamento de alta precisão a partir de sinais GNSS (Global Navigation Satellite System). O desafio na utilização de tal medida é a estimação da ambiguidade de fase. Para aplicações em tempo real, a estimação da ambiguidade deve ser feita com a menor quantidade de dados possíveis, mas isso pode influenciar na precisão da determinação das baselines e, portanto, da atitude. A distância entre as antenas do receptor também afeta a precisão da estimação da atitude. Os resultados deste trabalho demonstram a influência do comprimento das baselines na determinação da atitude em tempo real.

Palavras-chave - Navegação, Ambiguidade de fase, Baseline.

\section{Introdução}

O posicionamento GNSS pode ser obtido a partir da determinação das distâncias entre os receptores que compõem o sistema multiantena. O cálculo de tais distâncias, chamadas de baselines, pode ser efetuado com acurácia utilizando a medidas da fase da onda portadora. No entanto, isto requer a estimação correta da quantidade de ciclos inteiros propagados entre o satélite e o receptor. Tal medida é também conhecida como ambiguidade de fase (Park and Kim, 1998; Ribeiro, 2011).

No posicionamento GNSS em tempo real, a ambiguidade precisa ser solucionada no menor tempo possível. No entanto, a utilização de poucas medidas pode causar erros na estimação das ambiguidades e, consequentemente, influenciar na acurácia da determinação da atitude GNSS (Chen et al., 2011).

Por não existir uma solução analítica para o problema em questão, diversas técnicas para a estimação da ambiguidade de fase têm sido propostas, como: Least Squares Ambiguity Decorrelation Adjustment (LAMBDA) (Teunissen, 1994) e Mixed Integer Least Squares (MILES) (Chang et al., 2005).

Um outro fator que pode afetar a determinação da atitude GNSS é a distância entre as antenas dos receptores, ou seja, o comprimento das baselines. A influência da ambiguidade de fase, assim como do comprimento das baselines, na determinação da atitude tem sido bastante discutida (Reis et al., 2012; Chen et al., 2011; Carvalho, 2012).

Neste trabalho é verificada experimental- mente a relação entre o comprimento das baselines e o erro na sua estimação, além de se investigar a influência de tal erro na determinação da atitude GNSS. Os métodos utilizados na obteção dos resultados são apresentados na Seção 2. Os resultados são discutidos na Seção 3 e a Seção 4 conclui o trabalho.

\section{Metodologia}

O cálculo do posicionamento GNSS está sujeito a diversas fontes de erro. Um meio efetivo de minimiza-los é utilizar o conceito de posicionamento diferencial. O posicionamento diferencial utiliza as medidas da fase da portadora para estimar as baselines e, em seguida, utiliza as mesmas para determinar a posição do corpo. A utilização da pseudodistância também permite o posicionamento GNSS, no entanto sem a mesma acurácia.

O posicionamento diferencial consiste em um receptor de referência, ou mestre, cuja localização é conhecida, e um ou mais receptores embarcados no corpo do qual prentende-se obter a localização, também conhecidos como escravos (Carvalho, 2012). A Figura 1 ilustra esse conceito.

\subsection{Modelagem matemática}

O posicionamento diferencial parte do princípio de que duas antenas próximas estão sujeitas aos mesmos erros do sinal transmitido por um determinado satélite. A diferença entre esses sinais recebidos simultaneamente por dois receptores pode minimizar o erros provenientes da propagação do 


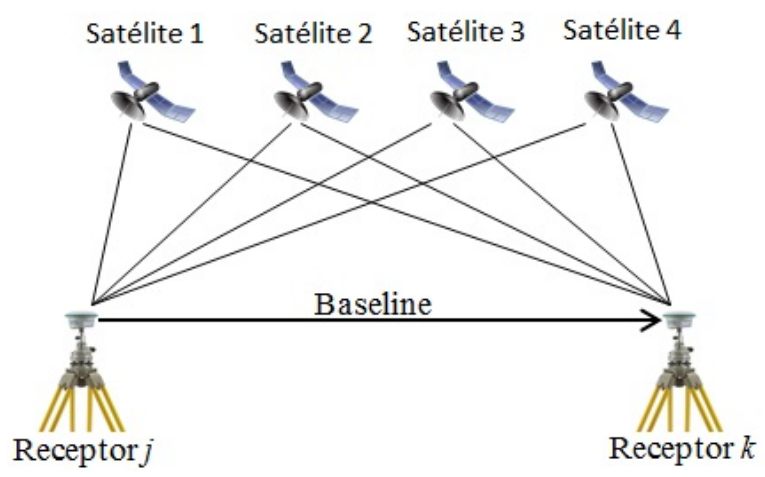

Figura 1: Conceito de posicionamento diferencial.

sinal e do sincronismo dos relógios dos satélites. Tal procedimento é chamado de diferença simples de fase.

A realização da diferença entre duas diferenças simples pode minimizar ainda os erros de relógio causados pelos receptores. A diferença dupla de fase, como é conhecida essa técnica, é geralmente calculada em relação ao satélite mais visível e à antena de referência. A equação da diferença dupla de fase é expressa por (Hofmann-Wellenhof et al., 1994):

$$
\phi_{j k}^{12}=\frac{1}{\lambda} H_{j k}^{12} \delta x+N_{j k}^{12}+\epsilon
$$

onde,

$\phi_{j k}^{12}$ - diferença dupla entre a fase da onda portadora observada pelos receptores j e k e transmitidas pelos satélites 1 e 2 ;

$\lambda$ - comprimento de onda da portadora;

$H_{j k}^{12}$ - matriz sensibilidade das baselines;

$\delta x$ - são as coordenadas da baseline;

$N_{j k}^{12}$ - diferença dupla da ambiguidade de fase da onda portadora observada pelos receptores j e k e transmitidas pelos satélites 1 e 2;

$\epsilon$ - ruído de medida da fase da onda portadora.

Este cálculo é realizado para todos os $m$ satélites visíveis, de forma que $\phi_{j k}^{12} \in \mathbb{R}^{m x 1}, H_{j k}^{12} \in$ $\mathbb{R}^{m x 3}$ e $N_{j k}^{12} \in \mathbb{R}^{m x 1}$. Seguindo o mesmo conceito, é possível também fazer a diferença dupla das medidas de pseudodistância, resultando

$$
\rho_{j k}^{12}=\frac{1}{\lambda} H_{j k}^{12} \delta x+\epsilon
$$

onde, $\rho_{j k}^{12}$ - diferença dupla entre a pseudodistância observada pelos receptores $\mathrm{j}$ e $\mathrm{k}$ e transmitidas pelos satélites 1 e 2 .

Apesar de (2) não possuir relação com a ambiguidade de fase, a característica do código $\mathrm{C} / \mathrm{A}$ é muito menos ruídosa que da fase da onda portadora. Por esse motivo, a combinação das medidas de pseudodistância com a fase da portadora possibilita maior precisão na determinação das ambiguidades de fase (Reis et al., 2012).

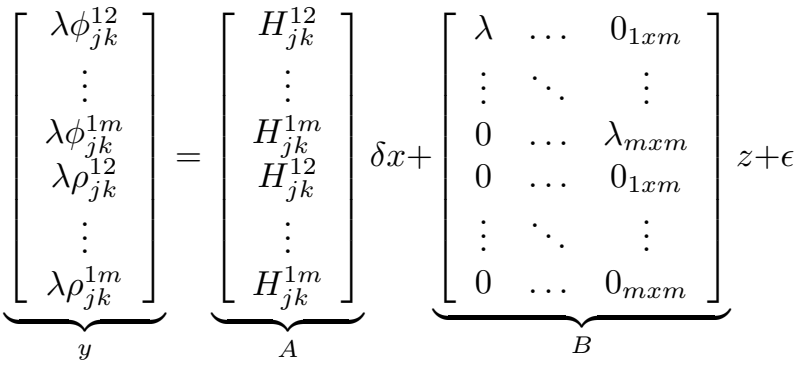

$\operatorname{com} z=\left[\begin{array}{c}N_{j k}^{12} \\ \vdots \\ N_{j k}^{1 m}\end{array}\right], \delta x=\left[\begin{array}{c}\Delta X \\ \Delta Y \\ \Delta Z\end{array}\right]$

Combinando as medidas de pelo menos quatro satélites, é possível estimar a ambiguidade de fase. Contudo, como apresentado na Seção 3, a utilização de várias épocas aumenta a acurácia na estimação das ambiguidades e das baselines, como esperado, devido à suavização do ruído.

\subsection{MILES}

O método MILES (Chang et al., 2005) é uma modificação do método LAMBDA (Teunissen, 1994). Ambos os métodos solucionam o problema da ambiguidade de fase, mas o MILES oferece melhor desempenho computacional (Chang and Zhou, 2011).

Sejam as matrizes $A \in \mathbb{R}^{m x k}, B \in \mathbb{R}^{m x n}$ e $y \in$ $\mathbb{R}^{m}$, suponha que $[A, B]$ possua posto completo. Então o algoritmo produz $p$ soluções ótimas para o problema MILS (Mixed Integer Least Squares)

$$
\min _{x \in \mathbb{R}^{k}, \mathbb{Z}^{n}}\|y-A x-B z\|_{2}^{2},
$$

no sentido que o par $\left\{x^{j}, z^{j}\right\} \in \mathbb{R}^{k}$ x $\mathbb{Z}^{n}$ é a jésima solução ótima se o resíduo correspondente à $\|y-A x-B z\|_{2}$ é o j-ésimo menor. Dessa maneira,

$$
\begin{aligned}
\left\|y-A x^{(1)}-B z^{(1)}\right\|_{2} & \leq \ldots \leq\left\|y-A x^{(j)}-B z^{(j)}\right\|_{2} \\
& \leq \ldots \leq\left\|y-A x^{(p)}-B z^{(p)}\right\|_{2} .
\end{aligned}
$$

onde o parâmetro $p$ é escolhido aleatoriamente de acordo com a quantidade de soluções ótimas a serem encontradas.

Nos casos em que não existir a matriz $A$, a Equação 4 torna-se um problema de mínimos quadrados inteiros, ou, Integer Least Square (ILS) .

$$
\min _{\mathbb{Z}^{n}}\|y-B z\|_{2}^{2} .
$$

Para solucionar o problema exposto em (4), primeiro é preciso separá-lo em dois problemas: um ILS e em um sistema de equações lineares triangular superior real. A solução destes dois subproblemas em sequência soluciona o MILS.

Suponha a fatoração QR de $A$

$$
A=\left[\begin{array}{ll}
Q_{A} & \bar{Q}_{A}
\end{array}\right]\left[\begin{array}{c}
R_{A} \\
0
\end{array}\right]
$$


onde $\left[Q_{A} \bar{Q}_{A}\right] \in \mathbb{R}^{m x m}$ é ortogonal e $R_{A} \in$ $\mathbb{R}^{m x m}$ é uma matriz superior triangular não singular. Esta fatoração pode ser computada pela transformação de Householder. Então,

$$
\begin{array}{r}
\|y-A x-B z\|_{2}^{2}= \\
\left\|\left[\begin{array}{c}
Q_{A}^{T} \\
\bar{Q}_{A}^{T}
\end{array}\right] y-\left[\begin{array}{c}
R_{A} \\
0
\end{array}\right] x-\left[\begin{array}{c}
Q_{A}^{T} B \\
\bar{Q}_{A}^{T} B
\end{array}\right] z\right\|_{2}^{2}= \\
\left\|Q_{A}^{T} y-Q_{A}^{T} B z-R^{A} x\right\|_{2}^{2}+\left\|\bar{Q}_{A}^{T} y-\bar{Q}_{A}^{T} B z\right\|_{2}^{2}
\end{array}
$$

Ao determinar um valor fixo para $z$, pode-se escolher um valor para $x$ de modo que o primeiro termo do lado direito da Equação 8 seja igual a zero. Portanto, para solucionar o MILS, primeiro é preciso estimar o valor de $\hat{z} \in \mathbb{Z}^{n}$ solucionando o ILS,

$$
\min _{\mathbb{Z}^{n}}\left\|\bar{Q}_{A}^{T} y-\bar{Q}_{A}^{T} B z\right\|_{2}^{2}
$$

e em seguida resolver o sistema triangular superior

$$
R^{A} x=Q_{A}^{T} y-Q_{A}^{T} B z
$$

para determinar a estimativa de $\hat{x} \in \mathbb{R}^{k}$. Assim, solucionar o MILS passa, obrigatoriamente, pela solução do ILS, cujo os detalhes podem ser observados em (Chang and Zhou, 2011).

\subsection{Determinação da atitude}

Uma vez estimadas as ambiguidades de fase, é possível estimar as baselines. Com as baselines estimadas, a determinação da atitude do corpo se restringe a um problema geométrico e pode ser realizada pelo método direto.

Para que os parâmetros da atitude possam ser computados, é preciso ter bem definido o sistema de coordenadas no qual o problema foi solucionado. Usualmente, as baselines são estimadas no ECEF (Earth Centered Earth Fixed) e, portanto, precisam ser transformadas para o sistema de navegação local, com a origem na antena mestre. $\mathrm{O}$ sistema de coordenadas utilizado no presente trabalho foi o ENU (East North Up).

Definidas as coordenadas das antenas 1 e 2 no ENU, o ângulo de roll e determinado utilizando-se as coordenadas das antenas 2 na equação (11).

$$
r=-\tan ^{-1}\left(\frac{z_{2}}{x_{2}}\right)
$$

Os ângulos de pitch e yaw, por sua vez, são determinados utilizando-se as coordenadas da antena 1 nas equações (12) e (13), respectivamente.

$$
\begin{gathered}
p=-\tan ^{-1}\left(\frac{z_{1}}{\sqrt{x_{1}^{2}+y_{1}^{2}}}\right) \\
w=-\tan ^{-1}\left(\frac{x_{1}}{y_{1}}\right)
\end{gathered}
$$

Convém notar que o método de computação direta da atitude requer apenas duas baselines simultaneamente.

\section{Resultados}

Foram utilizados quatro conjuntos de dados no presente trabalho. O primeiro foi extraído da toolbox de Determinação de Atitude com GPS (Dai et al., 2008). Tais dados foram gerados a partir do simulador IFEN NavX®e de 4 receptores Nova Tel®DL-4. Os demais conjuntos foram gerados a partir do primeiro, que por ser simulado, dispõe dos valores reais de posicionamento dos satélites e antenas, assim como das ambiguidades de fase.

A partir de tais informações foram geradas novas diferenças duplas de fase da portadora e de pseudodistância, alterando o comprimento das $b a$ selines e a intensidade do ruído. As próximas duas subseções apresentam os resultados das simulações com os dados originais e em seguida com os dados gerados.

\subsection{Dados originais}

Antes de apresentar a discussão dos resultados é preciso salientar o cenário favorável no qual os algoritmos foram simulados. Os dados disponíveis em (Dai et al., 2008) tratam de um sistema estático e com baselines relativamente longas, como pode ser observado na Figura 2, e com 9 satélites visíveis durante todas as épocas.

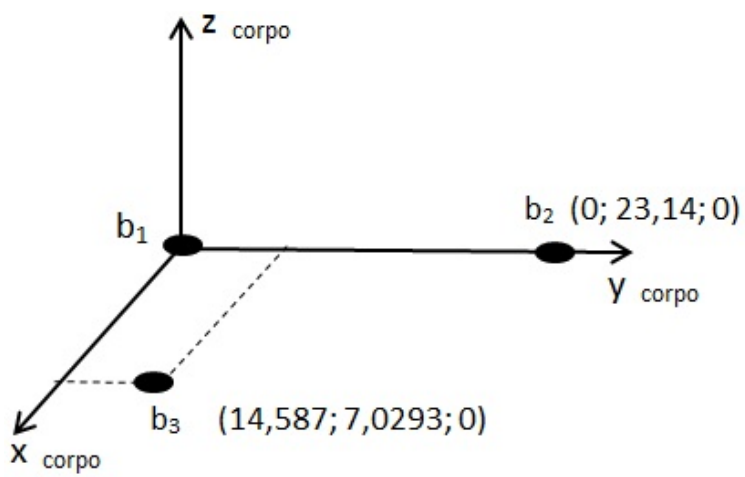

Figura 2: Posicionamento das antenas

Os sinais simulados em (Dai et al., 2008) são gerados utilizando o sistema de posicionamento relativo. Contudo, o sistema não possui uma referência fixa em terra, o que torna necessária a utilização de uma das antenas do corpo como referência. Nesse caso, é utilizado um algoritmo de posiciomanamento por ponto preciso de forma a obter-se a localização da antena principal. Tal procedimento degrada, em poucos centímetros, o posicionamento das antenas escravas e deteriora a estimativa das baselines.

As ambiguidades de fase são estimadas solucionando-se (6) e utilizando 10 épocas na janela deslizante. Uma vez estimadas as ambiguidades, (4) estima o comprimento das baselines. Os erros na estimação das baselines, apresentadas nas Figuras 3 e 4, se propagam na estimação da atitude. 


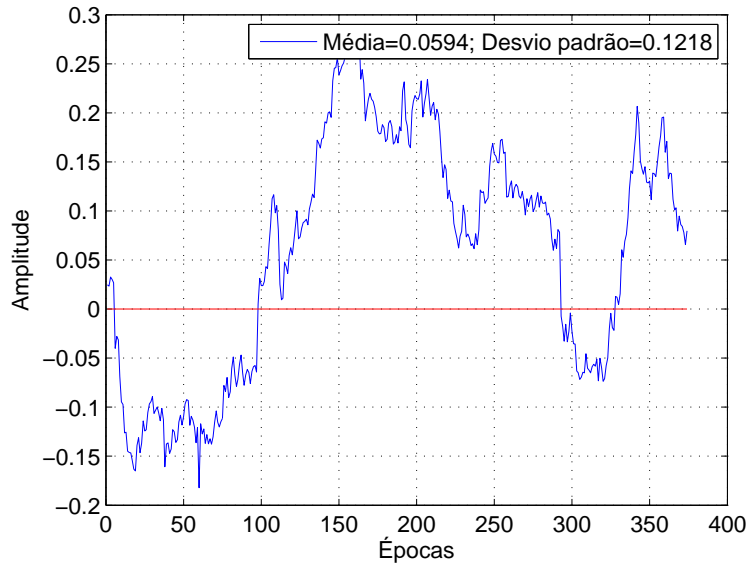

Figura 3: Erro da baseline 1-2 estimada

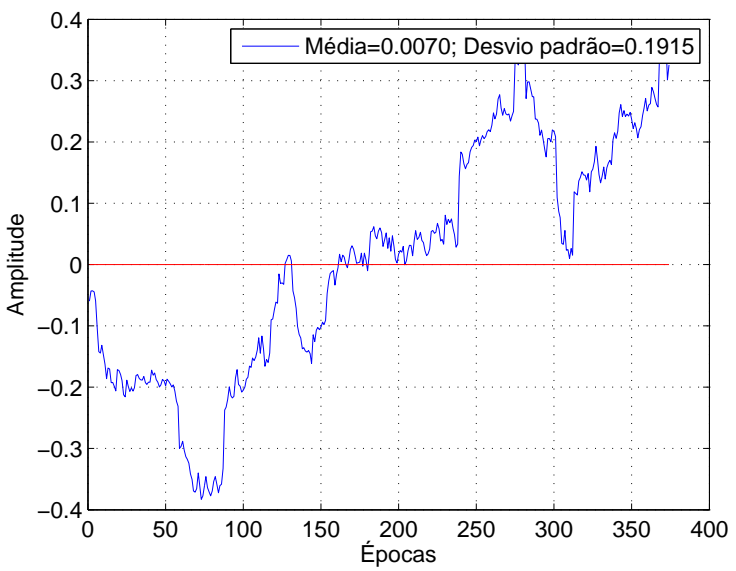

Figura 4: Erro da baseline 1-3 estimada

Comparando-se os erros de estimação das baselines 1-2 e 1-3, pode-se observar que, na média o erro da segunda é menor. No entanto, o desvio padrão mostra que, pontualmente, o erro na estimação da baseline 1-3 é maior. Tal comportamento pode ser justificado pela intensidade do ruido utilizado na geração dos dados.

Solucionada a ambiguidade de fase, as coordenadas das antenas escravas são determinadas e, em seguida, as equações (11), (12) e (13) determinam a atitude do corpo. A figura 5 apresenta os erros na determinação dos ângulos de roll, pitch e yaw.

\subsection{Dados gerados}

Os dados utilizados nesta parte das simulações, como dito anteriormente, foram derivados dos presentes em (Dai et al., 2008). De posse do posicionamento real dos satélites, das antenas e das ambiguidades de fase, foram gerados 3 novos conjuntos de dados, cada um com 4 combinações diferentes de baselines. Em cada um dos conjuntos foram utilizados ruídos de diferentes intensidades.
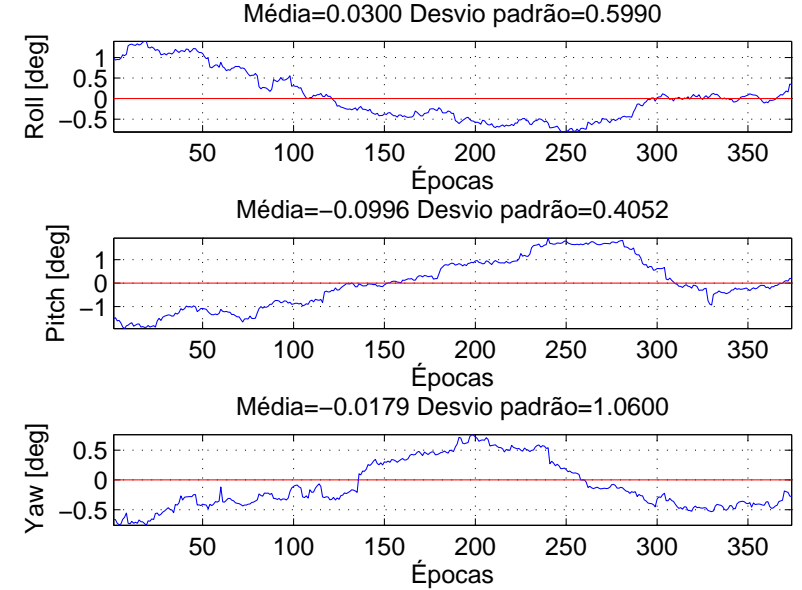

Figura 5: Erro da baseline estimada

No primeiro conjunto, as variâncias dos ruídos são iguais aos apresentados nas leituras dos sinais em (Dai et al., 2008). No segundo conjunto, a intensidade das variâncias foram duplicadas. No terceiro, as variâncias foram multiplicadas por 5 .

Na Tabela 1 são apresentados os comprimentos das baselines em cada uma das 4 configurações utilizadas.

Tabela 1: Comprimento das baselines em cada uma das configurações $(\mathrm{m})$

\begin{tabular}{c|cc}
\hline Baseline & $1-2$ & $1-3$ \\
\hline Configuração & & \\
1 & 23,1381 & 16,1579 \\
2 & 18,5266 & 13,5414 \\
3 & 13,8949 & 6,7707 \\
4 & 6,4843 & 4,0624 \\
\hline
\end{tabular}

A Tabela 2 mostra em quanto o comprimento das baselines foram reduzidas de uma configuração para a outra.

Tabela 2: Taxa de redução do comprimento das baselines em cada uma das configurações (\%)

\begin{tabular}{c|cc}
\hline Baseline & $1-2$ & $1-3$ \\
\hline Configuração & & \\
$1-2$ & 20 & 16,4 \\
$2-3$ & 25 & 50 \\
$3-4$ & 46,67 & 40 \\
\hline
\end{tabular}

As Tabelas 3 e 4 apresentam, respectivamente, as médias dos erros de estimação das $b a$ selines 1-2 e 1-3 para diferentes comprimentos e intensidades de ruído.

Pode-se observar que nem sempre uma baseline mais curta implica um maior erro relativo na sua estimação. Tal comportamento fica evidenciado nas Tabelas 5 e 6 , que apresentam como o erro na estimação do comprimento das baselines 1-2 e 
Tabela 3: Erros relativos na estimação das baselines $1-2(\%)$

\begin{tabular}{c|ccc}
\hline Intensidade ruído & 1 & 2 & 5 \\
\hline Configuração baselines & & & \\
1 & 0,24 & 0,50 & 0,81 \\
2 & 0,29 & 0,59 & 0,86 \\
3 & 0,37 & 0,74 & 0,81 \\
4 & 0,68 & 1,09 & 1,53 \\
\hline
\end{tabular}

Tabela 4: Erros relativos na estimação das baselines $1-3(\%)$

\begin{tabular}{c|ccc}
\hline Intensidade ruído & 1 & 2 & 5 \\
\hline Configuração baselines & & & \\
1 & 1,35 & 3,27 & 7,43 \\
2 & 1,61 & 3,89 & 8,81 \\
3 & 3,19 & 7,69 & 16,83 \\
4 & 5,26 & 12,58 & 25,84 \\
\hline
\end{tabular}

1-3, respectivamente, evolue entre uma configuração e outra.

Tabela 5: Evolução dos erros na estimação da $b a-$ seline $1-2(\%)$

\begin{tabular}{c|ccc}
\hline Intensidade ruído & 1 & 2 & 5 \\
\hline Conf. baselines & & & \\
$1-2$ & 20 & 18 & 6,17 \\
$2-3$ & 27,6 & 25,47 & $-5,84$ \\
$3-4$ & 83,78 & 47,3 & 88,8 \\
\hline
\end{tabular}

Tabela 6: Evolução dos erros na estimação da $b a-$ seline 1-3 (\%)

\begin{tabular}{c|ccc}
\hline Intensidade ruído & 1 & 2 & 5 \\
\hline Conf. baselines & & & \\
$1-2$ & 19,26 & 18,96 & 18,57 \\
$2-3$ & 98,14 & 97,69 & 91,03 \\
$3-4$ & 64,9 & 63,59 & 53,53 \\
\hline
\end{tabular}

Comparando as Tabelas 5 e 6 com a Tabela 2 fica claro que não existe uma relação direta entre o encurtamento da baseline e o erro na sua estimação. Comportamento semelhante é observado ao se inserir um ruído de maior intensidade no sinal recebido pelo receptor.

A seguir as Tabelas 7, 8 e 9 apresentam as médias dos erros de estimação dos ângulos de roll, pitch e yaw, respectivamente, para cada um dos conjuntos utilizados, empregando as baselines estimadas.

Como pode-se verificar nas Tabelas 7, 8 e 9, a estimação da atitude piora à medida que as baselines são encurtadas. O mesmo acontece quando aumenta-se o ruído. A piora mais acentuada é observada no ângulo de pitch, por utilizar todas as coordenadas da antena 1, como descrito em (12). O ângulo de roll utiliza as coordenadas da antena 2, que possui os maiores erros de estimação.
Tabela 7: Erros de estimação de Roll (graus)

\begin{tabular}{c|ccc}
\hline Intensidade ruído & 1 & 2 & 5 \\
\hline Conf. baselines & & & \\
1 & 0,3612 & 0,5134 & 1,3332 \\
2 & 0,4267 & 0,6251 & 1,7052 \\
3 & 0,5383 & 0,8213 & 2,3822 \\
4 & 1,0828 & 1,8651 & 6,1954 \\
\hline
\end{tabular}

Tabela 8: Erros de estimação de Pitch (graus)

\begin{tabular}{|c|c|c|c|}
\hline Intensidade ruído & 1 & 2 & 5 \\
\hline Conf. baselines & & & \\
\hline 1 & 1,5203 & 2,8158 & 7,2302 \\
\hline 2 & 1,8345 & 3,4252 & 8,8236 \\
\hline 3 & 2,9979 & 5,6547 & 15,2221 \\
\hline 4 & 5,5037 & 10,6165 & 29,4588 \\
\hline
\end{tabular}

Tabela 9: Erros de estimação de Yaw (graus)

\begin{tabular}{c|ccc}
\hline Intensidade ruído & 1 & 2 & 5 \\
\hline Conf. baselines & & & \\
1 & 1,4083 & 2,8158 & 7,1268 \\
2 & 1,7336 & 3,5608 & 8,8499 \\
3 & 2,2768 & 4,7109 & 11,6911 \\
4 & 4,7480 & 9,9131 & 23,7753 \\
\hline
\end{tabular}

\section{Conclusões}

Este trabalho investigou a influência do comprimento das baselines nos erros da sua estimação e na estimação da atitude. Para possibilitar tal análise, foram utilizadas diferentes configurações de comprimento das baselines, além da inserção de diferentes intensidades de ruído no sinal recebido pelos receptores.

Ficou evidenciado no estudo que a estimação do comprimento das baselines não é afetado pelo seu real comprimento.

Por outro lado, a estimação da atitude possui relação direta, com o comprimento das baselines. Tal conclusão se deve ao fato de que o método direto de determinação de atitude depende, conforme (11), (12) e (13), da proporção entre distâncias contaminadas por erros de mesma magnitude. Assim, os erros são menos significativos quanto maior o comprimento das baselines.

\section{Agradecimentos}

Os autores agradecem ao CNPq pelo suporte financeiro.

\section{Referências}

Carvalho, L. D. (2012). RIBEIRO, Lucas de Carvalho . Determinação ao da Atitude Utilizando Receptores GPS. 
Chang, X. W., Yang, X. and Zhou, T. (2005). MLAMBDA: a modified LAMBDA method for integer least-squares estimation, Journal of Geodesy 79(9): 552-565. DOI: $10.1007 / \mathrm{s} 00190-005-0004-\mathrm{X}$

Chang, X.-w. and Zhou, T. (2011). MILES : MATLAB package for solving Mixed Integer LEast Squares problems Theory and Algorithms, (January).

Chen, A., Zheng, D., Ramanandan, A., Farrell, J. A. and Measurements, A. D. (2011). Nearreal-time GPS Integer Ambiguity Resolution, pp. $7281-7286$.

Dai, Z., Knedlik, S. and Loffeld, O. (2008). Toolbox for GPS-based attitude determination : An implementation aspect, pp. 389-405.

Hofmann-Wellenhof, B., Lichtenegger, H. and Collins, J. (1994). GPS theory and practice, 3 edn, Springer-Verlag.

Park, C. and Kim, I. (1998). Integer ambiguity resolution for GPS based attitude determination system, Proceedings of the 37th SICE Annual Conference. International Session Papers pp. 1115-1120.

Reis, J. a., Sanguino, J. and Rodrigues, A. (2012). Baseline Influence on Single-Frequency GPS Precise Heading Estimation, Wireless Personal Communications 64(1): 185-196. DOI: 10.1007/s11277-012-0525-6

Ribeiro, L. D. C. (2011). Gnss ambiguity resolution with least squares methods, Proceedings of the 21th Brazilian Congress of Mechanical Engineering. .

Teunissen, P. J. G. (1994). A New Method for Fast Carrier Phase Ambiguity Estimation The Carrier Phase Observation Equation, pp. 562-573. 